\title{
Detection of Structural Vibration-Induced Noises with Modal Analysis in Diesel Generators
}

\author{
Uğur Ölmez (iD, Nevra Bayhan² (iD, Hakan Doğan' (D), Murat Uysal' (iD \\ 'Teksan Generator, İstanbul, Turkey \\ ${ }^{2}$ Department of Electrical and Electronics Engineering, İstanbul University-Cerrahpaşa, İstanbul, Turkey
}

Cite this article as: Ölmez U, Bayhan N, Doğan H, Uysal M. Detection of Structural Vibration-Induced Noises with Modal Analysis in Diesel Generators. Electrica, 2019; 19(1): 72-84.

\begin{abstract}
Diesel generators are one of the most common used energy sources for maintaining the continuity of electrical energy. Diesel generators cause high noises during their operation. There are many methods of eliminating the noise problems that diesel generators generate during energy production. However, these methods show differences according to the type of noise source. In this study, the problem of noise was emphasized that it is caused by structural vibrations of diesel generators. Therefore, the method of coherence function, which is an analytical method, was used for the detection of noise caused by structural vibrations. A test software and user interface was developed to perform sound and vibration measurement and analysis with using coherence function. The coherence analysis of sound and vibration data was made by performing various tests and measurements with the developed test software. In the coherence tests of diesel generator, the vibration frequencies that cause high noise at the measurement points were determined. In order to verify the coherence tests, the computer-aided 3D solid model of diesel generator used in the tests was created. Computer-aided modal analysis of the diesel generator was performed by using this model. The modal analysis was performed at the vibration measurement points and the frequencies that cause high noise. Coherence test results and modal analysis results were compared. It was seen that the results of the coherence measurement and the results of the computer-aided modal analysis supported each other. As a result of these studies, it was shown by computer-aided modal analysis that the high noise occurring in diesel generators can be determined by the coherence tests.
\end{abstract}

Keywords: Diesel generator, coherence, sound, vibration, modal analysis

\section{Corresponding Author: \\ Nevra Bayhan}

\section{E-mail:}

nevra@istanbul.edu.tr

Received: 12.12 .2018

Accepted: 26.12 .2018

(c) Copyright 2019 by Electrica

Available online at

http://electrica.istanbul.edu.tr

DOI: 10.26650/electrica.2019.18048

\section{Introduction}

Today, electrical energy has entered into every aspect of our lives in parallel with the rapid development of technology. The use of electrical energy in many areas such as hospitals, data centers, telecommunication base stations, industrial facilities has reached critical levels. In these areas, an instantaneous energy interruptions can result in massive catastrophes that end in loss of life and property.

Diesel generators are widely used to maintain the continuity of the electric energy. Diesel generators which detect electricity interruptions quickly and connect to the mains in a very short time are one of the most preferred energy production sources in back-up of grid.

The problem of noise caused by the widespread use of diesel generators is becoming a more important problem especially in the living areas such as housing, hospitals and business centers. For this reason, methods of reducing noise levels of the generators to the lower values have been investigated. The first thing to do in these studies is to determine the noise sources.

Diesel generators have many noise sources that cause high noise. The main sources that give rise to noise in diesel generators are classified as engine block noise, radiator fan noise, alternator mechanical noise, alternator induction noise, exhaust noise and structural vibration-induced noises.

The equipments of the diesel generator consisting of rotating parts such as engine, alternator and radiator cause the structural vibrations to occur on the chassis where the generator is 
fixed and surface of the sound attenuated enclosure connected to it. These structural vibrations cause the noise propagation from the chassis and sound attenuated enclosure surfaces. The main purpose of this study is to determine the vibration points and frequencies that cause noise on the generator chassis and sound attenuated enclosure.

At the beginning of this study, in order to determine the noises caused by the structural vibrations on the generator chassis and sound attenuated enclosure surfaces, the frequency analysis was applied to the noise and vibration data separately. Fourier Transform was used for frequency analysis of noise and vibration data. Fourier transform is an analytical method which is used to characterize linear systems and defines frequency components that create a continuous wave form [1].

Cooley and Tukey [2] developed an algorithm in 1965 that had the ability to calculate Fourier coefficients much faster than was needed in the past. This method is known as Fast Fourier Transform (FFT) nowadays. Frequency band analysis of noise and vibration data was performed by using FFT method.

As a result of studies which are done separately in the frequency band, there cannot be found direct relationship between noise and vibration data. For this reason, the coherence function was used to determine the relationship between sound and vibration data. The coherence function is defined as the counterpart of the normalized cross-correlation of time signals in the frequency domain.

The need for additional understanding of the noise generation process was enabled many studies on diesel engines at Herrick Laboratories. With the help of the obtained data from the noise studies that multiple input - single output (MISO) coherence models of diesel engines were created $[3,4]$.

In some studies on diesel engines, the coherence function has been used to determine the noise caused by cylinder pressures. Leclere et al. [5] performed a study to determine the relationship between cylinder pressure and noise. In this study, the relationship between vibration of cylinder pressure and the noise was investigated by coherence function. Narayan [6] has determined the relationship between the vibration of the cylinder pressures and the noise of diesel engines with the help of the coherence function. As a result of this study, Narayan [6] stated that engine noise control could be performed by controlling the injection parameters. Lamula et al. [7] examined the effect of different fuels such as diesel and gas on cylinder pressure. The effect of the vibrations which caused by the different cylinder pressures on the noise generation were obtained by the tests using the coherence function by Lamula et al. [7].

It is also possible to formulate in the time domain a MISO model which was used in some acoustic measurement applications by Goff [8] and also known as the correlation technique. Although, this method successfully has been applied in different work areas such as turbulence studies, it could not become widespread in noise control studies. However, Kumar and Srivastava [9] used this technique to get successful results for noise source identification of diesel engines.

Vibration-induced noise analysis with the coherence function has also been realized in different areas besides diesel engine applications. Pazara et al. [10] used the coherence analysis between noise and vibration to detect defects due to the production of bearings and other moving parts. Lukic [11] in his study, in order to analyse the noise and vibration problems which caused by hydraulic systems in the vehicles examined the sound pressure level and vibration levels of hydraulic pumps by using the coherence function.

In this study, the computer-aided 3D solid model of diesel generator used in the tests was created to verify the coherence tests. Computer-aided modal analysis of the diesel generator was performed by using this model. Modal analysis is the study of the dynamic properties of linear structures based on structural tests or simulaton-based finite element analysis. These dynamic properties include natural frequencies and structural modes. The dynamic properties depend on the mass, stiffness and damping distribution on the structure and determine the structural vibration behaviour when exposed to operational loads.

When linear structures exposed to a vibration at natural frequency values, they are resonated. This situation may cause to increase the amplitude of the vibration which the structure is exposed and cause permanent damages to the structure. Therefore, it is important to determine the natural frequency values of the structures. The natural frequency values of the structures can be determined by modal analysis method and necessary precautions can be taken.

Several studies have been carried out to determine the natural frequency values of various equipment of diesel generator. The majority of these studies have been performed for diesel engines which are the main power source of the generators. These studies have generally been concentrated on the crankshaft and cylinder block of diesel engines. In addition, it was also observed that modal analysis studies were performed for various equipments such as chassis and control panel of diesel generators.

Carrato and Fu [12] performed a study to determine the torsional vibrations on the crankshaft of diesel engines. Carrato and Fu used a method which based on modal analysis techniques in their study. Meng et al. [13] performed the stress and modal analysis of the crankshaft of a 4-cylinder diesel engine with the finite element method. Meng et al. [13] analysed the vibration modes, impact and stress conditions of the crankshaft in their study. With the results of analysis, they obtained information about optimization and improvement of diesel engine design.

Espador et al. [14] conducted a study to determine the cause of the breakage after the cylinder and liner break in a diesel 
engine of a 7.5 MW (MegaWatt) generator which is operating in a power plant. It was seen that the critical areas which are determined by the finite element analysis of the liner match the fracture zones of the liner. Jiang and Yan [15] investigated the results of modal analysis with different mesh densities of a diesel engine block. The results which are obtained from the study constitute a reference for element selection in future modal analysis studies. The results also provide the basis for structural dynamic analysis and noise control studies in future studies.

Aher and Malave [16] conducted a study to understand how the excitation forces from engine and alternator mounting feet propagates to the rest of the generator structure. In addition to the study, modal analysis was also performed to determine the potential modes and natural frequencies of the diesel generator set. In the results of modal analysis, it was observed that several modes were close to motor harmonics and these modes could increase the vibration response of the generator set. Magdum and Yadav [17] examined the mechanical properties of the material which is used in the diesel engine test bed on the natural frequency, mode shape and frequency response of the test bed. In the study, the effect of two materials of different densities on the natural frequency and mode shape was investigated. Magdum and Yadav [17] concluded that the mechanical properties of the used material had a direct effect on the natural frequency and mode shape. Kadam and Zope [18] in their study discussed the design that will optimize the vibrations that the control panel of a diesel generator set can be resonated.

This study was performed to contribute to the literature studies to determine the relationship between the noise and vibration of diesel generators. Therefore, it was aimed to determine the noise which generated by the vibrations on the chassis and sound attenuated enclosure of diesel generators based on the coherence studies on diesel engines. Also in this study, a computer aided modal analysis study was performed to determine the natural frequency values of the diesel generator chassis and sound attenuated enclosure. The aim of this study to compare the vibration values which are obtained by the coherence analysis with the natural frequency values which are obtained from the computer aided modal analysis results.

\section{Material and Method}

In order to perform the coherence analysis of diesel generators, a test software was developed which can perform sound and vibration measurement and give the coherence values of the measurement for this experimental study. A user interface was also designed for this software. A test setup was established in order to perform coherence analysis and various tests were carried out with the developed software. In the tests, coherence analysis were performed by being taken sound and vibration measurements from different points of the generator [19].
In this study, unlike [19], modal analysis method was applied for the chassis and sound attenuated enclosure of the diesel generator. Natural frequency values of the chassis and sound attenuated enclosure parts of the generator were found by modal analysis method. The natural frequency values calculated by the modal analysis were compared with the structural vibration-induced noise points which are obtained in the tests.

\section{Coherence function analysis}

The mathematical method that uses determining the relationship between two or more signals is called coherence analysis. The normalized cross correlation of two analog signals in the time domain is defined as coherence in the frequency domain.

Single Input - Single Output (SISO) coherence model was used in this study. Figure 1 shows a block diagram which belongs to SISO coherence model.

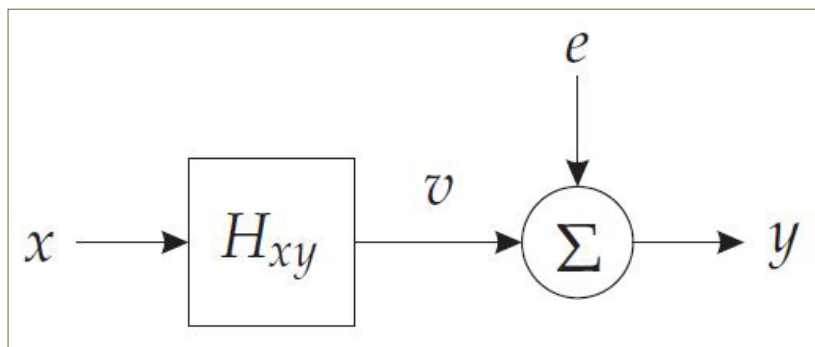

Figure 1. A block diagram of single input - single output coherence model [20]

Since the coherence technique is defined as the frequency domain equivalent of the normalized cross correlation of the analog signals, the self spectral power densities and cross-spectral power densities of the analog signals are used in the coherence calculations. The cross-spectral power density calculation is given as

$$
S_{x y}=\frac{1}{K U} \sum_{k=1}^{K} X_{k}^{*} Y_{k}
$$

where $S_{x y}$ expresses the cross-spectral power density between the $x$ and $y$ signals, $K$ expresses element number and $X_{k}^{*}$ expresses complex conjugate of the signal $x$. The quantity constant $U$ is defined as

$$
U=f_{s} \sum_{n=1}^{M} W(n)^{2}
$$

where $W(n)$ is a constant that takes into account the spectral weight of the data window. $W(n)$ is used to properly scale the spectral power density, assuming that the input is measured as volts. $M$ is the element length corresponding to the windowing length. $f_{s}$ is the sampling rate. 
The self spectral power density value $S_{x x}$ of $x$ signal is calculating by typing $x$ instead of $y$ in the equation specified in (2). The method of calculating the self spectral power density $S_{x x}$ is given as

$$
S_{x x}=\frac{1}{K U} \sum_{k=1}^{K}\left|X_{k}\right|^{2}
$$

The transfer function of the input and output signals which is given by Figure 1 can be expressed by

$$
Y(f)=H(f) X(f)
$$

where $H(f)$ is the expression of the system transfer function. The system transfer function $H(f)$ as spectral power density is given as

$$
H(f)=\frac{S_{x y}(f)}{S_{x x}(f)}
$$

The coherence function is expressed by using cross and self spectral power densities and given as

$$
C_{x y}=\frac{\left|S_{x y}\right|^{2}}{S_{x x} S_{y y}}
$$

where $C_{x y}$ expresses to the coherence function between the two signals, $S_{y y}$ expresses to the self sprectral power density of signal $y$. Equation (6) is a mathematical expression in which the linear relationship between $x$ and $y$ signals is scaled between 0 and 1. If the result of this function is 0 or close to 0 , the $x$ and $y$ signals are unrelated; however, if the result of this function is 1 or close to 1 , the $x$ and $y$ signals are linearly related [20].

\section{Modal analysis method}

The behavior of the mechanical equipments under dynamic loads is determined using dynamic parameters to have characteristic feature for each mechanical component. These dynamic parameters are obtained by analytical models or experimental studies. Analytical modeling is called as modal analysis in the literature. Natural frequency values and mode shapes of the mechanical structure are obtained by modal analysis. The natural frequency is the frequency that it depends only on elasticity and mass of an object and when it is stimulated, it vibrates at high amplitude continuously. The mode shape refers to the shape and direction of displacement occurring when a structure vibrates at its natural frequency. In short, the mode shape is an expression that gives the direction and amplitude of the vibration. In this study, natural frequencies and mode shapes of the diesel generator chassis and sound attenuated enclosure were determined by modal analysis.

For the analysis of mechanical systems, at first, a mathematical model which can express the system adequately is created.
The mathematical model of mechanical systems is classified according to the degree of freedom and the state of damping. The degree of freedom is the number of coordinates which are required to define the position of each part of the mechanical system at any time. In this study, modal analysis calculations were explained on the mathematical model of a mechanical structure with single degree of freedom, undamped, free vibration. The mathematical model of an object with an undamped and single degree of freedom system was shown in Figure 2 [21].

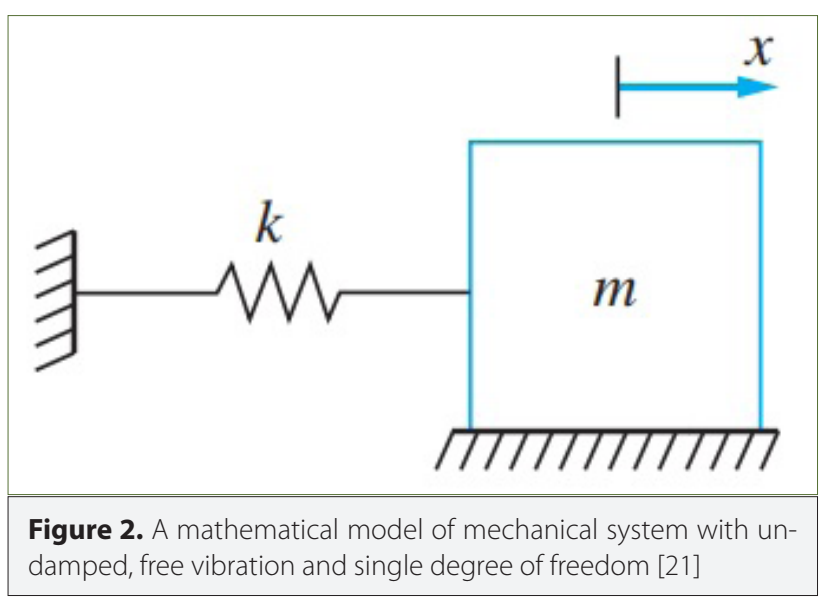

The equation of motion of an undamped, single degree of freedom mechanical system is given as

$$
M \ddot{X}(t)+K X(t)=0
$$

where $M$ is the mass of the mechanical structure and $K$ is the elasticity coefficient of the mechanical structure. The $X(t)$ refers to the quantity of displacement of vibration motion. The $X(t)$ which is used in (7) is generally expressed as

$$
X(t)=\phi \sin (w t+\varphi)
$$

where $\varphi$ is the amplitude vector, $w$ is the frequency value, $\varnothing$ is the phase angle. If derivative of (8) is taken twice, the expression is obtained as

$$
\ddot{X}(t)=-w^{2} \phi \sin (w t+\varphi)
$$

Substituting (9) and (8) into (7) and rearranging it results in

$$
\left(K-w^{2} M\right) \phi=0
$$

Equation (10) is in the format of numerical eigenvalue problem. The solution of this problem is found by equalizing the determinant of the coefficient matrix to zero. The mathematical expression of the coefficient matrix is shown as

$$
\left|K-w^{2} M\right|=0
$$

The solution of (11) causes the formation of $\mathrm{n}$ different roots with degree of $w^{2}$. The roots obtained in the form of $w_{1}^{2}, w_{2}^{2}$ 
$, \ldots, w_{n}^{2}$ are expressed as the eigenvalues of the equation of motion. These obtained eigenvalues are also natural frequencies of the mechanical structure. There is a $\mathrm{n}$ dimensional $\phi_{i}$ eigenvector corresponding to each $w_{i}^{2}$ eigenvalue. $\phi_{i}$ eigenvectors are obtained by solving the expression given by

$$
\left(K-w_{i}^{2} M\right) \phi_{i}=0
$$

These obtained eigenvectors are also called mode shape or natural mode of the mechanical structure. The mode shape is a vector which expresses the direction of vibration of the structure. A mathematical expression of eigenvectors is given as [22].

$$
\phi_{i}=\left\{\phi_{1 i}, \phi_{2 i}, \ldots, \phi_{n i}\right\}^{T}
$$

\section{Software development for coherence analysis}

The coherence function was used to determine the relationship between the noise and vibration of the diesel generators. For this reason, a test software was developed which can perform sound and vibration measurements and also perform coherence analysis of these measurements.

In the first phase of the software development, signal measurement and processing stages were carried out. The measured sound and vibration signals with the aid of a microphone and accelerometer are transmitted to the data acquisition device. The analog signals which are measured by the data acquisition device are transformed into a digital signal by sampling. In this study, sampling was performed in "continuous" mode and 18 $\mathrm{kS} / \mathrm{s}$ sampling rate.
Following the coding studies related to signal reading stage, the signal processing phase was started such as FFT and coherence calculations. The codes for the calculation of the coherence function of the sound and vibration signals were written according to the equation which is given in (6) and the results were shown graphically in the frequency axis. In addition, the codes which are related to the FFT transformations of the sound and vibration signals were written separately and the results were shown graphically in the frequency axis. The codes which are related to the calculation of RMS (Root Mean Square) levels of sound and vibration signals were also written in the signal processing phase [23].

Fast Fourier Transform analysis of sound and vibration measurements was performed at $1500 \mathrm{~Hz}$ bandwidth. Because of the fact that the sound and vibration data of the diesel generators are concentrated at the low frequencies in the frequency axis, it was thought that $1500 \mathrm{~Hz}$ bandwidth will be sufficient.

Different analysis methods were added to the test software to be able to make more detailed sound and vibration analysis. With the help of these analysis methods, it is possible to perform octave band analyses of the sound measurement and the velocity and displacement analysis of the vibration measurement [23].

The software also includes sound recording capability in accordance with ISO 3744 standard in order to perform generator sound measurements [24]. A reporting interface that transfers measurement data to Excel was also added the software so that the measurement results can be backed up and reported.

Figure 3 shows a screenshot of the user interface of the developed software. In the right section of the software, there are

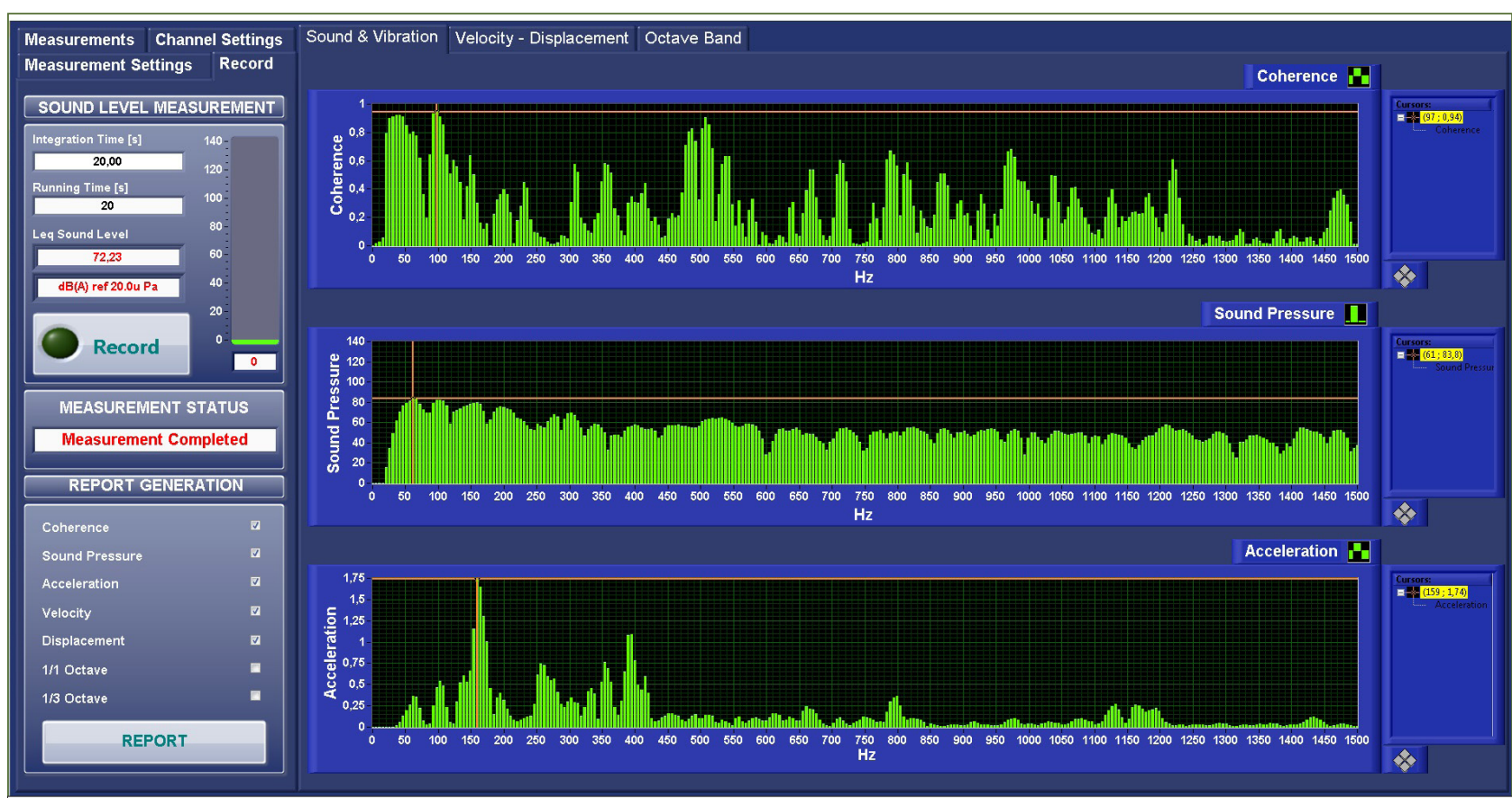

Figure 3. A screenshot of the user interface of the developed test software 
frequency band analysis of the coherence, sound and vibration measurements. In the left section, there are tabs for the settings and measurement results, including sound recording and reporting features.

\section{Experimental Results}

\section{Test setup}

In this study, a test setup was prepared in order to determine the noise problems of the diesel generators due to the structural vibrations. In the test setup, a 4-cylinder diesel generator which is at 21 kVA standby power and operates at a constant speed of $1500 \mathrm{rpm}$ was used. An acoustic microphone was used for generator noise measurements. An accelerometer was used for the vibration measurements. The sound and vibration signals were sampled using the data acquisition device and transferred to the test software. The obtained signals were analysed using the test software. An image of the test setup is shown in Figure 4.

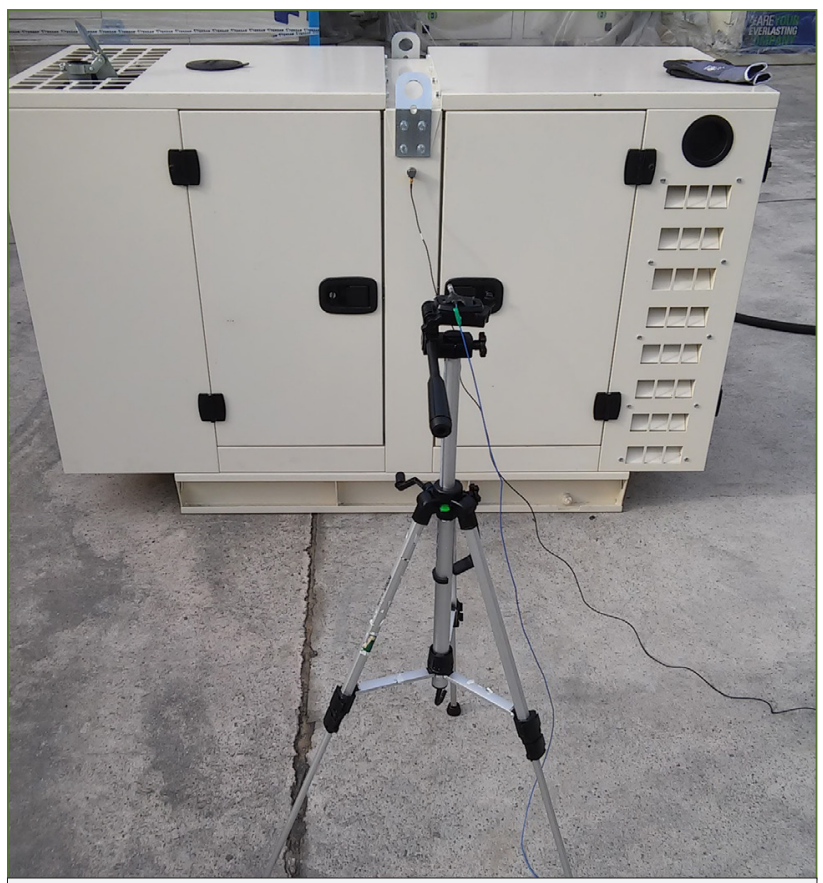

Figure 4. Test setup for coherence analysis

In this study, noise measurements were performed according to the indicated methods and reference measurement points in the ISO 8528-10 standard [25]. However, the noise measurements as well as the vibration measurements were also carried out in this study. For this reason, noise measurements were taken only for surfaces where vibration was measured. Because of the fact that the effect of the vibration on the noise was felt less felt at the corner points, noise measurements were not carried out at the corner points. Figure 5 shows the reference points for generator noise measurement. The noise measurements for this study were performed at reference points 1, 2, 3, 4 and 9 .

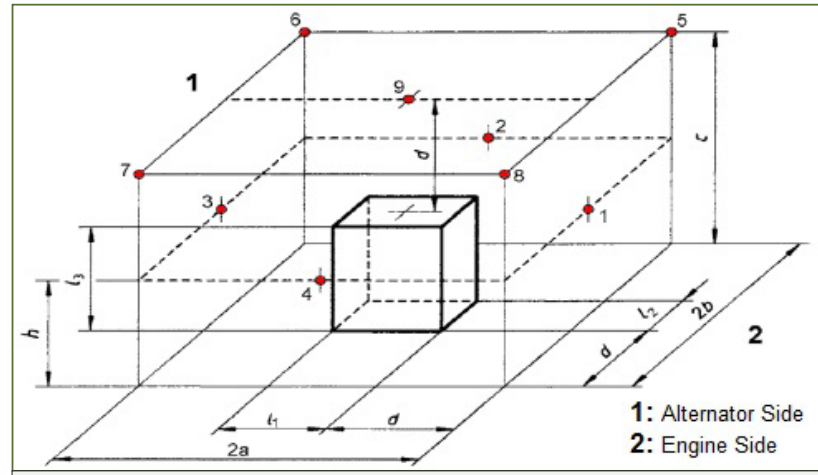

Figure 5. Reference measurement points used for noise measurements [25]

Noise measurements were made at a distance of 1 meter from the generator surface and the ground.

According to the ISO 8528-10 standard, the noise measurements of diesel generators are performed with power of $\% 75$ $\mathrm{kW}$ of the generator prime power. For this reason, noise measurements were performed at a load power of $11.4 \mathrm{~kW}$ which is $\% 75$ power of the diesel generator.

The vibrations of the generator chassis and sound attenuated enclosure surfaces are not known since any vibration measurements have not been taken before. Therefore, vibration measurements were taken from the center and end points of all parts on sound attenuated enclosure and chassis. Vibration measurements were taken from 59 different points including the front, rear, right, left and top surfaces of the generator sound attenuated enclosure and the top and side surfaces of the chassis. Figure 6 shows that an image of the reference points of vibration measurements taken from the sound attenuated enclosure and chassis.

\section{Test results}

Noise and vibration measurements were taken many points on the generator surfaces with using test setup and software. Noise and vibration measurements were made separately from each determined point on generator surfaces. Coherence analysis of the measured signals was carried out with the help of the developed test software. Frequency domain analysis of sound and vibration signals was performed separately using the test software and the frequency characteristic of the system was examined.

It was seen that only the coherence analysis was not sufficient to achieve a clear result. Therefore, frequency analysis of the sound and vibration values of the generator were included in the evaluation criteria. For this purpose, the velocity value of the vibration measurement according to the criteria specified in ISO 10816-1 standard was obtained and used to evaluate the results [26]. Here, the reference value for velocity magnitude was selected as $1.8 \mathrm{~mm} / \mathrm{s}$, which is the lower limit for small machines of Class-1 type specified in the ISO 10816-1 standard. In 

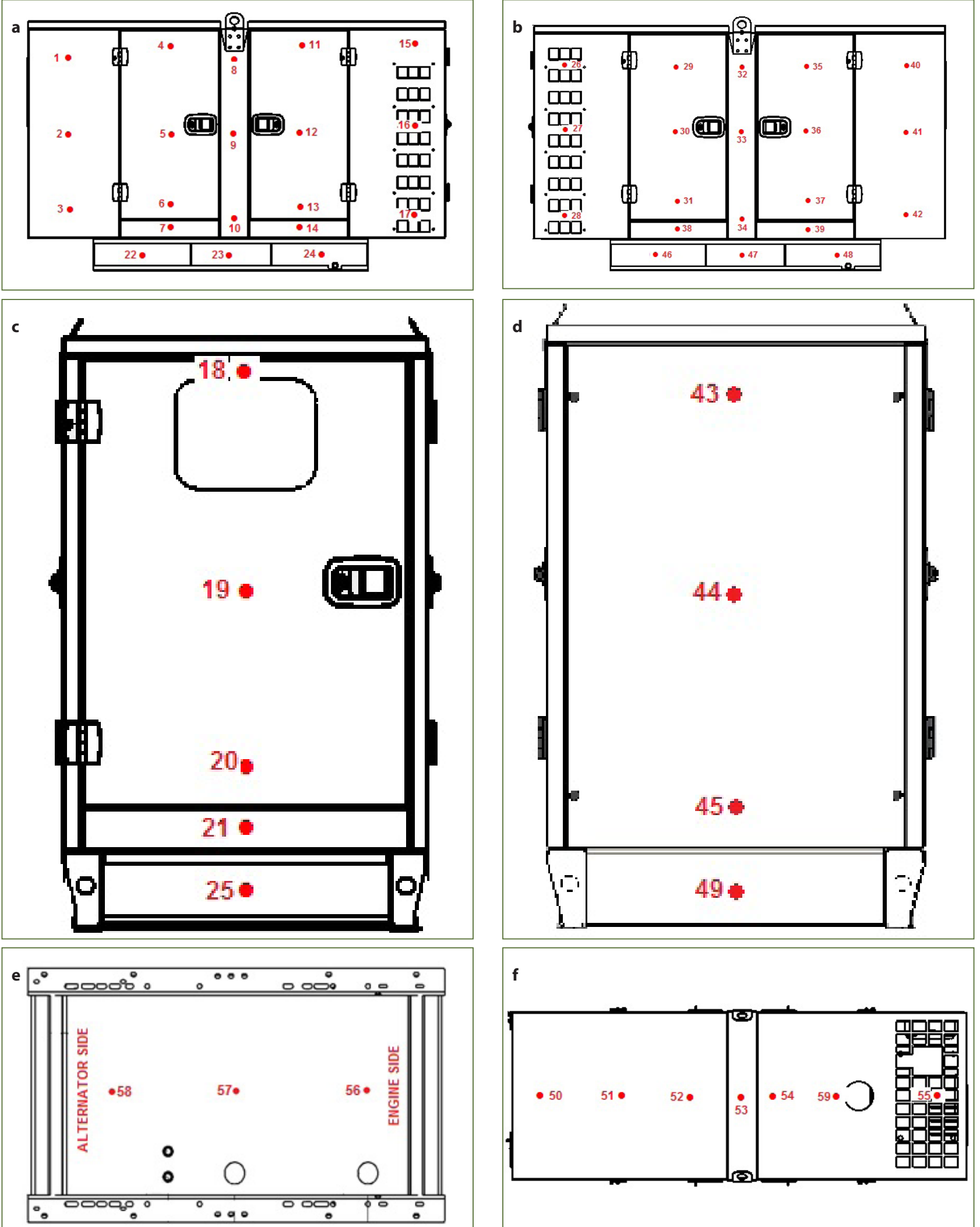

Figure 6. a-f. Reference measurement points for vibration measurements. From left side of generator (a). From right side of generator (b). From front side of generator (c). From back side of generator (d). From top side of chassis (e). From top side of generator ( $f$ ) 
this standart, machines up to $15 \mathrm{~kW}$ are considered as Class-1 machines.

The evaluation criterion for sound level measurement in this study is the octave band values of the measured sound levels on a frequency domain. The octave band values of the sound measurement were taken into account in the cases below 10 $\mathrm{dBA}$ of the Leq (Equivalent Continuous Sound Level) sound level of the same measuring points. The reason for selecting 10 $\mathrm{dBA}$ here is that the noise level difference between sources up to $10 \mathrm{dBA}$ affects the ambient sound level.

The most important criterion in the evaluation of the test results is the coherence analysis. For the frequencies which are below $4000 \mathrm{~Hz}$, the coherence limit is selected to 0.8 [27]. In this study, the coherence limit was chosen as 0.7 in order to be able to keep the evaluation scale slightly wider. However, it is already mentioned that only the coherence value is not sufficient to reach a clear result. Therefore, the octave band level and vibration velocity level was examined simultaneously for frequency values with high coherence value. The frequency values which have high octave sound level and high vibration velocity level were considered as frequency values that have noise problem related to vibration.

Figure 7 shows the measurement results for the vibration measurement point- 15 graphically. The reason for selecting measurement point- 15 is that it contains vibrations at the frequency of $150 \mathrm{~Hz}$, which is the $3^{\text {rd }}$ harmonic of the generator. All other measurement points were also measured in the same format. The graph shows the frequency curves of the coherence, sound pressure and vibration acceleration values. According to the graph given in Figure 7, it is observed that the frequency values such as $50 \mathrm{~Hz}, 100 \mathrm{~Hz}, 150 \mathrm{~Hz}, 200 \mathrm{~Hz}$ and 500 $\mathrm{Hz}$ have high coherence values. At the evaluation stage, the measured sound levels were converted from $d B$ to $d B A$. Since the dBA sound levels at $50 \mathrm{~Hz}, 200 \mathrm{~Hz}$ and $500 \mathrm{~Hz}$ were considerably lower than Leq sound level, noises at these frequencies were not taken into account.

As a result of the detailed examination of the test results, it was determined that vibration induced noise problems occurred on 13 different vibration measurement points on the sound attenuated enclosure and the chassis of the generator. According to test results, it was seen that the noise related to the vibration occured at $63 \mathrm{~Hz}, 100 \mathrm{~Hz}, 125 \mathrm{~Hz}$ and $160 \mathrm{~Hz}$ octave frequencies. The test results were shared in Table 1. The values given in the Table 1 are the results of noise and vibration analysis of the diesel generator selected for this study. According to these values, it is concluded that vibration induced noise is generated at the given vibration measurement points.

\section{Modal analysis results}

The field of the study in which the effect of mechanical equipment under dynamic loads is determined with the help of dynamic parameters called as modal analysis in the literature. Natural frequency values that can cause deformation in mechanical structure are determined by modal analysis. In order to perform modal analysis of diesel generator which is used in

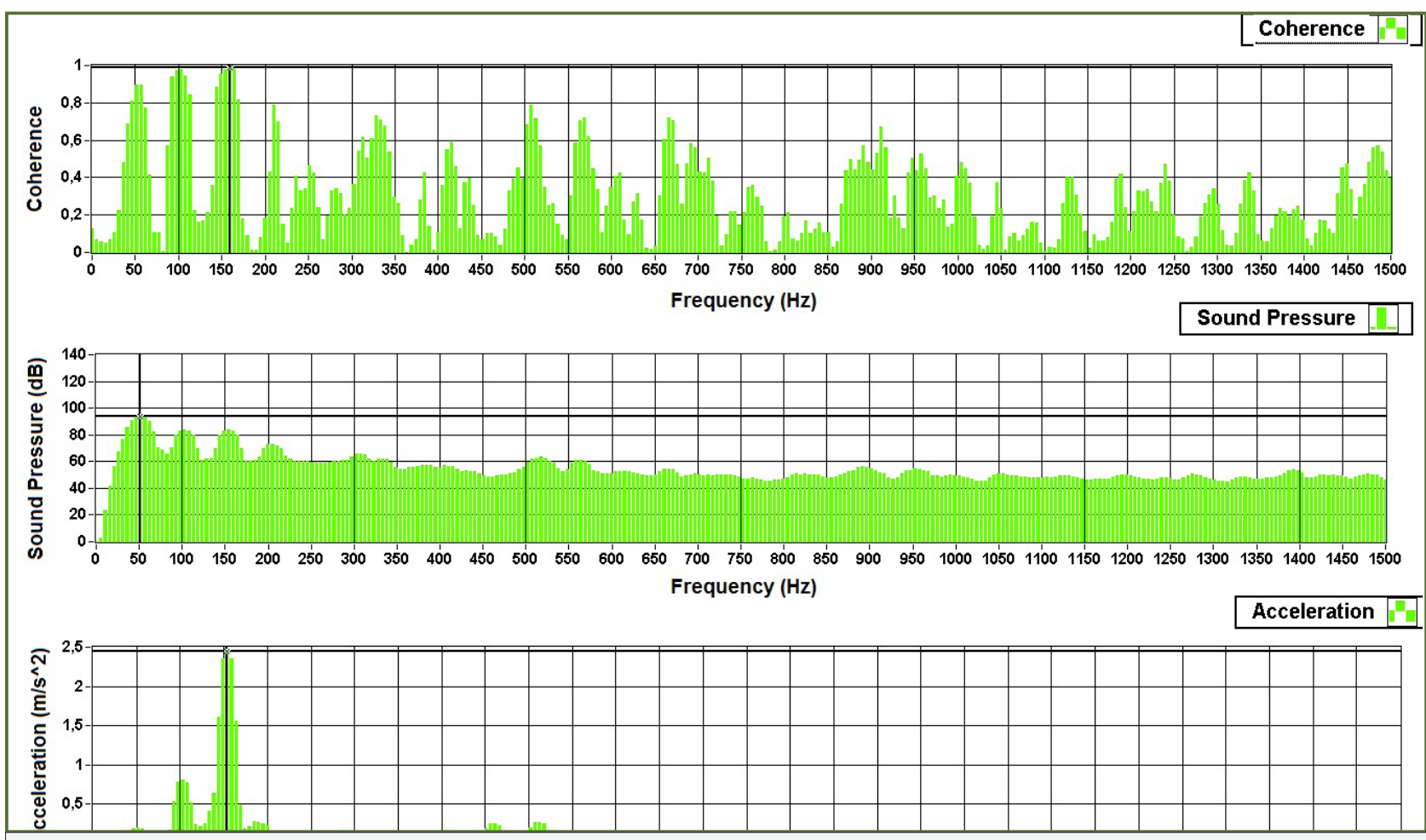

Figure 7. Graphical analysis of coherence, sound pressure and vibration acceleration values for measurement point-15 
Table 1. Vibration points that cause high noise level

\begin{tabular}{|c|c|c|c|c|c|}
\hline No & $\begin{array}{c}\text { Frequency } \\
\text { (Hz.) }\end{array}$ & $\begin{array}{c}\text { Coherence } \\
\text { Value }\end{array}$ & $\begin{array}{c}\text { Velocity } \\
\text { Value } \\
\text { (mm/s) }\end{array}$ & $\begin{array}{c}\text { Oktave } \\
\text { Sound } \\
\text { Level } \\
\text { (dBA) }\end{array}$ & $\begin{array}{l}\text { Leq. } \\
\text { Sound } \\
\text { Level } \\
\text { (dBA) }\end{array}$ \\
\hline \multirow{2}{*}{2} & 97.3 & 0.83 & 2.32 & \multirow{2}{*}{69.11} & \multirow{2}{*}{72.49} \\
\hline & 102.4 & 0.88 & 2.13 & & \\
\hline \multirow{5}{*}{3} & 92.2 & 0.91 & 3.35 & \multirow{4}{*}{66.44} & \multirow{5}{*}{72.25} \\
\hline & 97.3 & 0.9 & 6,33 & & \\
\hline & 102.4 & 0.86 & 8.03 & & \\
\hline & 107.5 & 0.8 & 8.64 & & \\
\hline & 122.9 & 0.71 & 2.55 & 63.58 & \\
\hline 4 & 61.4 & 0.9 & 1.82 & 62.02 & 72.96 \\
\hline \multirow{5}{*}{15} & 102.4 & 0.95 & 1.82 & 70.32 & \multirow{5}{*}{72.78} \\
\hline & 148.5 & 0.8 & 3.41 & \multirow{4}{*}{74.13} & \\
\hline & 153.6 & 0.89 & 3.37 & & \\
\hline & 158.7 & 0.92 & 3.07 & & \\
\hline & 163.8 & 0.87 & 2.05 & & \\
\hline 17 & 158.7 & 0.78 & 1.9 & 74.73 & 72.65 \\
\hline \multirow{4}{*}{18} & 92.2 & 0.95 & 2.16 & \multirow{4}{*}{65.93} & \multirow{4}{*}{73.45} \\
\hline & 97.3 & 0.95 & 3.24 & & \\
\hline & 102.4 & 0.9 & 3.5 & & \\
\hline & 107.5 & 0.79 & 3.26 & & \\
\hline 42 & 153.6 & 0.81 & 1.85 & 74.46 & 72.69 \\
\hline \multirow{3}{*}{50} & 97.3 & 0.9 & 2.09 & \multirow{3}{*}{66.96} & \multirow{3}{*}{73.43} \\
\hline & 102.4 & 0.92 & 2.45 & & \\
\hline & 107.5 & 0.9 & 2.53 & & \\
\hline \multirow{5}{*}{51} & 92.2 & 0.87 & 1.97 & \multirow{4}{*}{67.77} & \multirow{5}{*}{73.33} \\
\hline & 97.3 & 0.9 & 2.67 & & \\
\hline & 102.4 & 0.89 & 2.65 & & \\
\hline & 107.5 & 0.87 & 2.53 & & \\
\hline & 112.6 & 0.77 & 1.86 & 66.79 & \\
\hline \multirow{3}{*}{55} & 56.3 & 0.93 & 3.76 & \multirow{3}{*}{62.59} & \multirow{3}{*}{72.84} \\
\hline & 61.4 & 0.93 & 5.08 & & \\
\hline & 66.6 & 0.89 & 4.66 & & \\
\hline
\end{tabular}

\begin{tabular}{|c|c|c|c|c|c|}
\hline \multirow{6}{*}{57} & 61.4 & 0.82 & 2.27 & \multirow{2}{*}{63.31} & \multirow{6}{*}{73.8} \\
\hline & 66.6 & 0.79 & 2.25 & & \\
\hline & 92.2 & 0.76 & 2.95 & 68.32 & \\
\hline & 97.3 & 0.8 & 4.69 & & \\
\hline & 102.4 & 0.8 & 5.13 & & \\
\hline & 107.5 & 0.78 & 4.83 & & \\
\hline \multirow{5}{*}{58} & 92.2 & 0.81 & 3.17 & & \multirow{5}{*}{73.71} \\
\hline & 97.3 & 0.82 & 4.69 & & \\
\hline & 102.4 & 0.82 & 4.98 & & \\
\hline & 107.5 & 0.82 & 4.57 & 70.65 & \\
\hline & 112.6 & 0.76 & 2.92 & 67.04 & \\
\hline \multirow{7}{*}{59} & 61.4 & 0.71 & 2.36 & 63.18 & \multirow{7}{*}{73.69} \\
\hline & 92.2 & 0.82 & 1.89 & \multirow{4}{*}{69.92} & \\
\hline & 97.3 & 0.89 & 2.49 & & \\
\hline & 102,4 & 0.91 & 2.71 & & \\
\hline & 107.5 & 0.9 & 2.62 & & \\
\hline & 112.6 & 0.82 & 1.8 & 65.89 & \\
\hline & 148.5 & 0.72 & 1.91 & 70.34 & \\
\hline
\end{tabular}

Hz: hertz; mm/s: millimetre/second; dBA: a-weighted decibels; Leq: equivalent continuous sound level

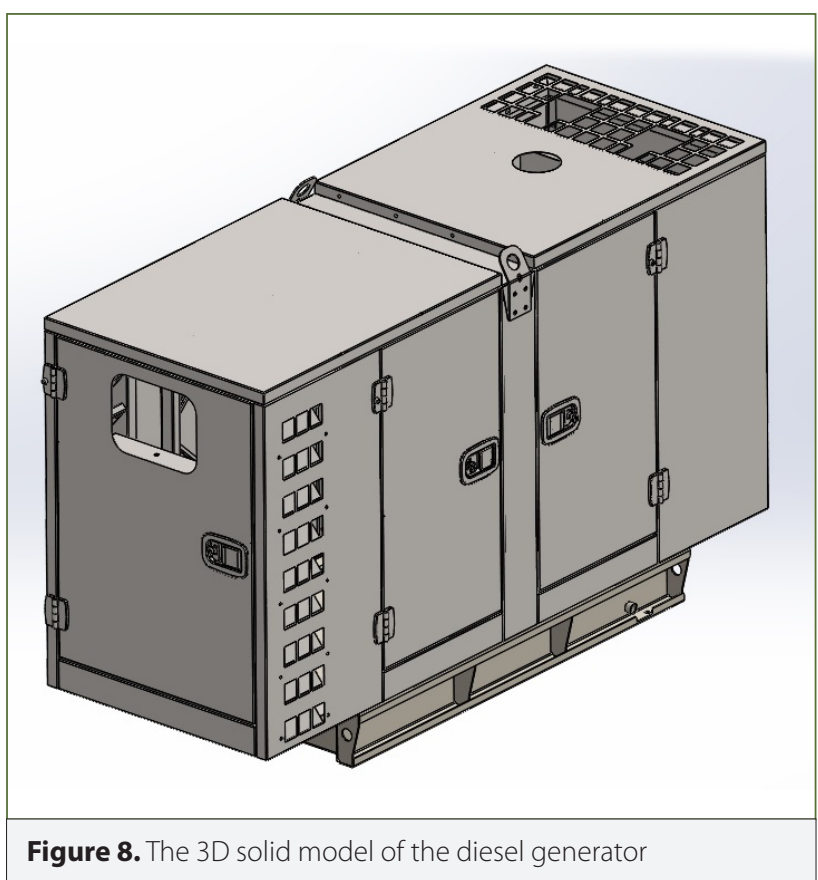


the tests, a computer-aided 3D solid model of the generator was prepared. The 3D solid model of the diesel generator was designed using the SOLIDWORKS software. S235JR type steel was used in the design of the solid model. The thickness of the material which is used in solid model is $1.5 \mathrm{~mm}$ on the sound attenuated enclosure surfaces and $3 \mathrm{~mm}$ on the chassis surfaces. The designed solid model is physically similar to the diesel generator which is used in the tests. Figure 8 shows an image of the $3 \mathrm{D}$ solid model of the diesel generator.

The 3D solid model which is designed to perform modal analysis was transferred to the ANSYS software. ANSYS software is an analysis software that uses the finite element method (FEM). Mesh operation of designed solid model were performed in ANSYS software. The mesh opration is expressed as the process of splitting a physical definition range into smaller definition ranges. The aim of meshing is to facilitate the solution of the differential equation to be solved. Therefore, the accuracy of the results to be obtained in the finite element method depends on the type of element and the number of elements which are used in the mesh.

The number of elements that are used in the mesh operation of the diesel generator is 421010 . The mesh element type which is used in the meshing is tetrahedron and the number of nodes is 923591 . Figure 9 shows an image of the mesh type of the diesel generator.

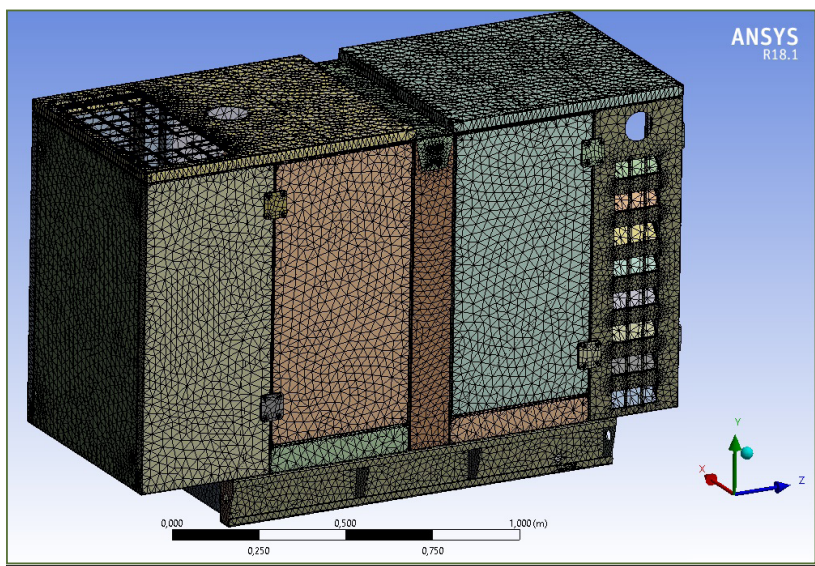

Figure 9. The mesh form of the diesel generator

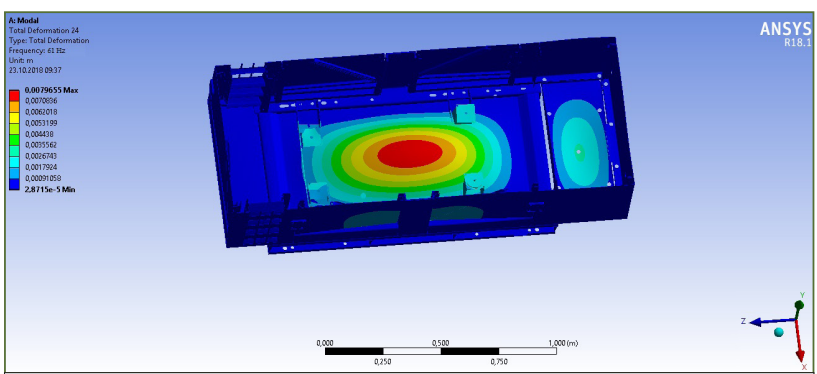

Figure 10. Modal analysis result, for the chassis upper surface at the frequency of $61 \mathrm{~Hz}$
The designed 3D solid model was subjected to modal analysis in a frequency range of $0-200 \mathrm{~Hz}$ according to a free and unforced motion equation. The system was examined in such a way that it was not exposed to external force and the natural frequency values and mode shapes of the structure were obtained.

In this paper, the vibration points which were found by experimental studies and caused high noise were examined by modal analysis for each vibration point and frequency value. As a result of the analysis, it was observed that the natural frequencies determined by the modal analysis have the same values as the vibration points determined by the experimental studies and they were performed at the same measuring points.

Figure 10,11 and 12 show the images which belongs to the modal analysis results. The modal analysis result of the chassis upper surface is shown in Figure 10. According to the results of the model analysis, it was seen that the vibration measurement point-57 on the chassis has a natural frequency at $61 \mathrm{~Hz}$. The mode shape is as shown in Figure 10. The vibration levels were scaled with colors in the blue-red range. The structures that vibrate at the same frequency with the natural frequency value reach the higher vibration levels with the resonance effect. With the performed tests, it was observed that the vibration values which are obtained from the measurement

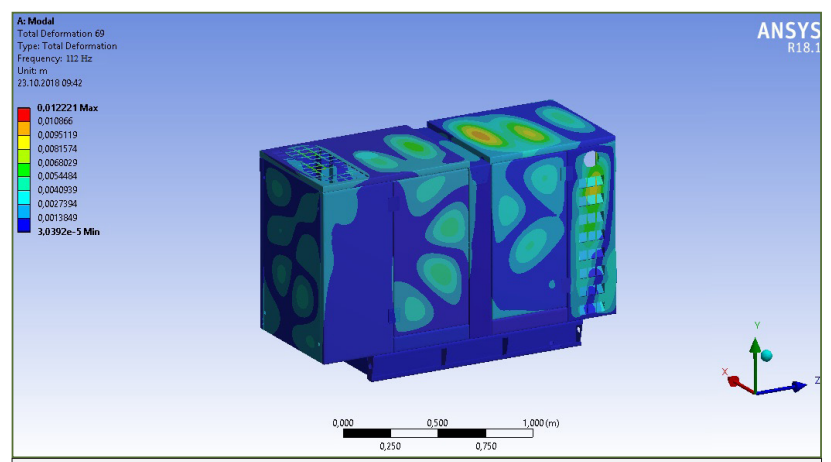

Figure 11. Modal analysis result, for the left and upper surface of sound attenuated enclosure at the frequency of $112 \mathrm{~Hz}$

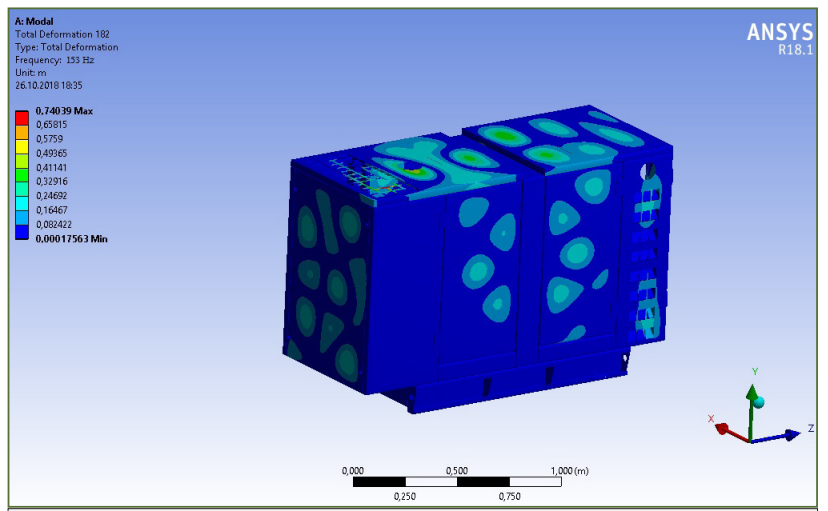

Figure 12. Modal analysis result, for the left and upper surface of sound attenuated enclosure at the frequency of $153 \mathrm{~Hz}$ 
point-57 were high. This high vibration level causes an increasing on the noise level of the measurement point-57. The noise and vibration values of the measurement point-57 are shown in Table 1.

The modal analysis result of the left and upper surfaces of the sound attenuated enclosure are shown in Figure 11. According to the results of the model analysis, it was seen that the vibration measurement point-51 on the upper surface of the sound attenuated enclosure has a natural frequency at $112 \mathrm{~Hz}$. The mode shape is as shown in Figure 11. With the performed tests, it was observed that the vibration values which are obtained from the measurement point-51 were high. This high vibration level causes an increasing on the noise level of the measurement point-51. The noise and vibration values of the measurement point-51 are shown in Table 1.

Similarly, the modal analysis result of the left and upper surfaces of the sound attenuated enclosure at $153 \mathrm{~Hz}$ are shown in Figure 12. According to the results of the model analysis, it was seen that the vibration measurement point-15 on the left surface of the sound attenuated enclosure has a natural frequency at $153 \mathrm{~Hz}$. With the performed tests, it was observed that the vibration values which are obtained from the measurement point-15 were high. This high vibration level causes an increasing on the noise level of the measurement point-15. The noise and vibration values of the measurement point- 15 are shown in Table 1.

According to the results of the modal analysis, it was seen that in all of the vibration points which are determined in Table 1, the mode shapes were formed related to the natural frequency at the determined frequencies. The obtained mode frequency in the analysis and the regions in which the mode shape occurring are consistent with the test results. According to this result, it is concluded that the computer aided modal analysis supports the results of the coherence tests for diesel generators.

As a result, modal analysis of the sound attenuated enclosure and the chassis surfaces of the diesel generator were made and compared with the results obtained from the tests. In the light of the data obtained, it was also supported by the modal analysis method that the diesel generator have noise problems caused by structural vibrations.

\section{Conclusion}

In this study, it was aimed to determine the noise problems related to the structural vibration of diesel generators. Therefore, a test software that can perform coherence analysis was developed. Furthermore, sound and vibration measurements were taken for the coherence analysis. In addition, a computer aided model of the generator was created and modal analysis were performed.

In the literature researches, the noises caused by the vibrations which formed with the combustion of the fuel in the diesel engines were investigated by using coherence function. The ex- amined studies are the papers on how the raw noise of diesel engines is affected by the noise generated by the vibrations on the engine surface. In this paper, it was investigated how the noise level measured from outside the sound attenuated enclosure of diesel engines was affected by the noise generated from the sound attenuated enclosure and the chassis vibrations. At the same time, the generator chassis and sound attenuated enclosure were subjected to modal analysis and it was aimed to find the vibration points and frequencies that may cause noise. Modal analysis and test results were compared and it was investigated whether the vibration points that cause noise support each other.

When the tests and analysis results were examined, it was observed that the diesel generator had noise problem at 2, 3, 4, $15,17,18,42,50,51,55,57,58$ and 59 numbered vibration measurement points. The noise problem at these vibration measurement points were observed at the octave frequencies of $63,100,125$ and $160 \mathrm{~Hz}$ respectively. As a result of this information;

It was seen that diesel generators have noise problems caused by structural vibrations.

It was concluded that the vibration-induced noise points can be determined by using the modal analysis results.

Whether the test points which are obtained by the modal analysis results generate vibration-induced noise was determined by coherence tests.

At the vibration points numbered 2, 3, 4, 15, 17, 18, 42, 50, 51, $55,57,58$ and 59 on the generator chassis and sound attenuated enclosure was seen that had noises related structural vibrations. These noises were seen at 63, 100, 125 and $160 \mathrm{~Hz}$ octave frequencies.

It was observed that the noise points related with vibration are generally concentrated in the cooling air inlets-outlets, on the doors, on the sound attenuated enclosure upper surfaces and also the chassis upper surfaces.

By using the data obtained as a result of this study, it is planned to reduce the determined noise problems in future studies. In this context, the studies planned to be carried out in the first step in order to reduce the level of structural vibration-induced noises are thought to be the selection of vibration dampers with better damping ability at the determined frequencies and increasing the wall thickness of the material to reduce the vibration levels at the vibration points that cause high noise.

Peer-review: Externally peer-reviewed.

Conflict of Interest: The authors have no conflicts of interest to declare.

Financial Disclosure: The authors declared that this study has received no financial support. 


\section{References}

1. K. B. Howell, "Fourier Series", in Principles of Fourier Analysis, Boca Raton, USA: Chapman \& Hall/CRC, 2001, ch. 2, pp. 93-242. [CrossRef]

2. J.W. Cooley, J. W. Tukey, "An algorithm for the machine calculation of complex fourier series", Mathematics of Computation, vol. 19, no. 90, pp. 297-301, Apr, 1965. [CrossRef]

3. M. J. Crocker, J. F. Hamilton, "Modelling of diesel engine noise using coherence", SAE Transactions, vol. 88, no 2, pp. 1263-1273, 1979.

4. M. J. Crocker, G. Anderkay, J. Y. Chung, "Controlling the noise radiated from diesel engines", Proceeding of First Interagency Symposium on Transportation Noise Research, California, USA, 28-30 March 1973, pp. 631-647.

5. Q. Leclere, J. Doruet, E. Parizet, "Extraction and analysis of diesel engine combustion noise", Proceedings of Exploratory Workshop on Modern Methods of Vibro-Acoustic Study with Application to Automative, Pitesti, Romania, 2011, pp. 37-43.

6. S. Narayan, "A review of diesel engine acoustics", FME Transactions, vol. 42, no. 2, pp. 150-154, 2014. [CrossRef]

7. L. Lamula, K. Saine, K. Saarinen, J. Hyrynen, "Cylinder pressure generated noise of medium speed diesel engine", Joint Baltic-Nordic Acoustics Meetings, Reykjavik, Iceland, 17-19 Aug, 2008.

8. K.W. Goff, "The application of correlation techniques to some acoustic measurements", J Acoust Soc Am, vol. 27, no. 2, pp. 336346, 1955. [CrossRef]

9. S. Kumar, N.S. Srivastava, "Investigation of noise due to structural vibrations using a cross-correlation technique", J Acoust Soc Am, vol. 57, no. 4, pp. 769-772, 1975. [CrossRef]

10. T. Pazara, M. Pricop, I. C. Scurtu, C. Pricop, O. Radu, "Defect identification of moving parts of a mechanical installation using correlation between vibration and noise", Scientific Bulletin of Naval Academy, vol. 19, no. 2, pp. 504-509, 2016.

11. J. Lukic, "An approach to an NVH investigation of vehicle hydrolic pumps", Journal of Low Frequency Noise Vibration and Active Control, vol. 30, no. 2, pp. 137-147, 2011. [CrossRef]

12. P. J. Carrato, C. C. Fu, "Modal analysis techniques for torsional vibration of diesel crankshafts", SAE Transactions, vol. 95, no. 4, pp. 955-963, 1986. [CrossRef]

13. J. Meng, Y. Liu, and R. Liu, "Finite element analysis of 4-cylinder diesel crankshaft", IJIGSP, vol. 3, no. 5, pp. 22-29, 2011. [CrossRef]

14. F. J. Espadafor, J. B. Villanueva, M. T. Garcia, E. C. Trujillo, "Analysis of a diesel generator cylinder failure", Engineering Failure Analysis, vol. 17, no. 4, pp. 913-925, 2010. [CrossRef]
15. S. Z. Jiang, W. B. Yan, "FEM modal analysis of diesel engine block", Advanced Materials Research, vol. 971-973, pp. 481-484, 2014. [CrossRef]

16. P. B. Aher, S. K. Malave, “Vibration assessment of diesel engine genset mounts", International Conference on Ideas, Impact and Innovation in Mechanical Engineering - ICIIIME 2017, Pune, India, 1-2 Jun, 2017, pp. 1700-1706.

17. S. Magdum, S. D. Yadav, "A finite element analysis of diesel engine test bed using different materials for reducing diesel engine bed vibrations", IJCEA, vol. 12, special issue, pp. 1-8, 2018.

18. A. R. Kadam, S. B. Zope, "Analysis of diesel generator control panel using finite element method", International Conference on Ideas, Impact and Innovation in Mechanical Engineering - ICIIIME 2017, Pure, India, 1-2 Jun, 2017, pp. 577-581.

19. U. Ölmez, H. Doğan, M. Uysal, "Noise analysis of diesel generators with coherence function", Irditech 2018 International R\&D, Innovation and Technology Management Congress, İstanbul, Turkey, 18 May, 2018, pp. 307-316.

20. T.C. Richards, "Dynamic testing of data acquisition channels using the multiple coherence function", in Data Acquisition Applications, Z. Karakehayov, Ed, London, United Kingdom: Intech Open, 2012, ch. 3, pp. 51-78.

21. S. G. Kelly, "Free vibrations of SDOF systems", in Mechanical Vibrations Theory and Applications, Si, SI ed., Stamford, USA: Cengage Learning, 2012, ch. 3, pp. 137-204.

22. Z. Q. Qu, "Theory of modal analysis," in Modal Order Reduction Techniques with Applications in Finite Element Analysis, London, United Kingdom: Springer-Verlag, 2004, ch. 3, pp. 31-46.

23. U. Ölmez, "Sound and vibration analysis of diesel generators with coherence function", M. S. thesis, Dept Electrical and Electronics Eng, İstanbul Univ, İstanbul, Turkey, 2018.

24. Acoustics - Determination of Sound Power Levels of Noise Sources Using Sound Pressure - Engineering Method in an Essentially Free Field over a Reflecting Plane, ISO 3744: 2010.

25. Reciprocating Internal Combustion Engine Driven Alternating Current Generating Sets - Part 10: Measurement of Airborne Noise by the Envoloping Surface Method, ISO 8528-10: 1998.

26. Mechanical Vibration - Evaluation of Machine Vibration by Measurements on Non-Rotating Parts - Part 1: General Guidelines, ISO 10816-1: 1995.

27. P. R. Donavan, D. M. Lodico, "Measuring tire-pavement noise at the source", Petaluma, CA, USA, NCHRP Report 630, 2009. [CrossRef] 


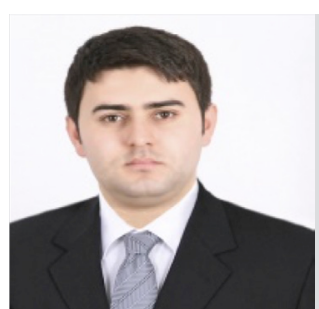

Uğur Ölmez was born in Istanbul on September 3, 1989. He received his B.Sc degree in Electrical and Electronic Engineering from Pamukkale University in 2013. He completed his M.Sc. degree in Electrical and Electronics Engineering from Istanbul University in 2018. Currently, he is working as a Senior R\&D Engineer in R\&D Center of Teksan Generator. His research interests are electric power generation, renewable energy technologies, genetic algorithm and signal processing.

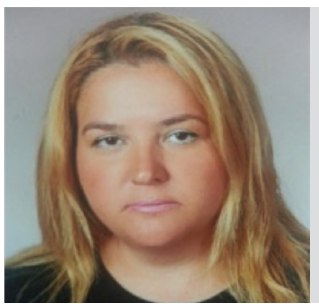

Nevra Bayhan was born in Istanbul. She received her B.Sc. and M.Sc. degrees in Electrical and Electronics Engineering from Istanbul University in 1997 and 2001, respectively. She completed her Ph.D. in Control and Automation Engineering at Istanbul Technical University in 2008. Since 2011, she has been working at the Electrical and Electronics Engineering Department of Istanbul University as an Assistant Professor. Her research interests are automatic control systems, control systems design, robust control, time-delay systems, digital control systems, energy, low order controller design and control of systems with parameter uncertainties.

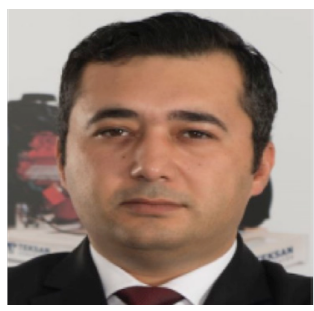

Hakan Doğan was born in Istanbul on October 28, 1978. He received his associate's degree in Electronics Department from Marmara University in 1998. He completed his B.Sc. degree in Mechanical Engineering at Ataturk University in 2002. Since 2014, he has been working at the R\&D Center of Teksan Generator as a R\&D Manager. His research interests are acoustic, vibration, CFD, FEM and energy.

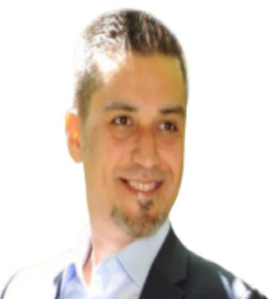

Murat Uysal was born in Izmir on April 25, 1983. He received his B.Sc. degree in Mechanical Engineering from Suleyman Demirel University in 2004. Currently, he is working at the R\&D Center of Teksan Generator as a R\&D chief. His research interests are passive type exhaust silencers, sound attenuated enclosures, FEM, vibration and airborne noise reduction techniques. 